\title{
ANALYTICAL STABILITY STUDY OF A DETERMINISTIC CAR FOLLOWING MODEL UNDER MULTIPLE DELAY INTERACTIONS
}

\author{
Rifat Sipahi $^{1} \quad$ Silviu-Iulian Niculescu \\ HeuDiaSyC (UMR CNRS 6599), Université de Technologie de \\ Compiègne, Centre de Recherche de Royallieu, BP 20529, \\ 60205, Compiègne, France.
}

\begin{abstract}
Analytical stability study of some deterministic car following models under time-delay influences is presented and various case studies are demonstrated. Interestingly, for some control law deployed by human drivers, more than one stability interval in the domain of time delay is revealed. Physical interpretations along with comparisons conclude the study.
\end{abstract}

Keywords: Car Following Model, Delay, Crossing Curves, Stability, Sensitivity

\section{INTRODUCTION}

Traffic flow problem has been one of the focus research topic for researchers since 1930s, for mathematical modeling see (Orosz, et al. 2004, Treiber a, et al. 2000, Bando, et al. 1996, Chandler, et al. 1958, Orosz, Stepan 2004), for empirical studies see (Hoogendoorn, Ossen 2005, Treiber a, et al. 2000, Kerner 1998) and for software development see (Helbing 2001). Among many parameters defining the traffic dynamics, there exists a critical one which is recognized in the traffic studies as early as in 1958s (Chandler, et al. 1958): it is the time delay. It mainly originates due to the time needed by human operators in sensing, decision making and acting against dynamical variations in the evolution of traffic flow. For this reason, traffic dynamics, and ultimately its mathematical models, inherently carry time delays. In this sense, see for instance (Orosz, et al. 2004, Sipahi,

\footnotetext{
1 Corresponding author: rsipahi@hds.utc.fr, Phone: +33.3.44.23.44.84, Fax: +33.3.44.23.44.77. The research of Dr. Sipahi is supported by Chateaubriand Post-Doctoral Bourse 2005-2006 of the French Government. Author is grateful for the hospitality he received from Dr. Martin Treiber and his research team during his visit at Technische Universität Dresden, funded by Centre National de la Recherche Scientifique (CNRS).
}

Niculescu 2006) for some models, related discussions and interpretations.

Time delay may drastically deteriorate the interpreted characterizations of the delay-free traffic models since its existence may not guarantee stability or instability. Therefore, a thorough stability analysis of the dynamics in time delay domain is needed in order to understand allowable time-delay margins of the human operators.

Origin of Time Delay: Before mentioning the mathematical models, we wish to introduce the modelling of time-delay in traffic flow dynamics. From (Sipahi, Niculescu 2006), we summarize the classification of time delay according to its origin in traffic dynamics. This is of importance as it presents how time delays should appear in mathematical models. Without going into details, the following three components form the time-delay, (inspired by (Bando, et al. 1996) and (Green 2000)):

- Physiological delay, $\bar{\tau}$ : (human operators), the period of time between receiving a stimulus and performing a control action. It appears as a pure time delay in mathematical models.

- Mechanical time delay, $\tilde{\tau}$ : period of time between the action of the driver and the time 
the vehicle responds. This time delay is following the physiological delay in an acceleration/deceleration action and it also appears as a pure time delay in mathematical models.

- Delay time of vehicle motion, $\bar{T}$ : not physically a pure time delay. It is defined as the period of time that a vehicle changes its velocity to the velocity of the preceding vehicle.

In this work we only focus on the time-invariant part of the delay and leave the treatment of time-varying and stochastic components of the delays for another study. For more detailed modelling of time delays, see (Hoogendoorn, Ossen 2005).

Quantitative Measure of Time Delay The time delays in the action of drivers are in the order of a second, although different ranges are stated in the literature, (Sipahi, Niculescu 2006, Green 2000). A delay of 0.75 - $1.0 \mathrm{sec}$, and a braking response time at the level of 1.2 - $1.35 \mathrm{sec}$ with a standard deviation of 0.6 $\mathrm{sec}$, is realistic. Moreover, sensing velocity variations need more time as pointed out in (Allen, et al. 2005): as a general remark, it is mentioned that response time of a human operator, when following a vehicle, decreases by $0.8 \mathrm{sec}$ for each increase in acceleration of the preceding vehicle by $0.3 \mathrm{~m} / \mathrm{sec}^{2}$. For a detailed discussion see (Sipahi, Niculescu 2006).

Once the delay modeling is established, mathematical models are needed for the stability analysis of the traffic flow dynamics. In this work, we particularly consider linear car following models, (Nagatani 1999, Nakayama, Sugiyama, et al. 2001, Rothery 1998, Treiber c, et al. 2005). Such an approach will allow some simplification in the model, but as we present below, time delay presence will induce further complications. The references mentioned above assume that the drivers create decisions with a timedelay, but following more than one vehicle in their decision-making offers larger stability margin in time delay domain. However, we wish to point out that by using a different model, an extended one from (Helbing 2001), some interesting conclusions can be arrived: one can suggest a more realistic model with multiple (non-identical) time delays for sensing the motion of different vehicles. The contribution of the present work is as follows:

1 We analyze the time-delayed traffic dynamics analytically via systems and control engineering perspective. Exact stability margins (maximum allowable time-delay for drivers) of the traffic flow when more than one preceding vehicle is followed, are presented on a low order dynamics.

2 A simple assumption is followed: a driver senses the motion of a nearer vehicle in front faster than sensing a vehicle further ahead. In other words, time delayed information obtained from different vehicles ahead of a vehicle are non-identical, i.e. independent from each other. This assumption is more realistic and it originates from the fact that drivers update their information more frequently from a nearer vehicle than from a further one.

To our best knowledge, an analytical study in the above perspective has not been pursued in the open literature and we form our main objective along this line. Our aim is also to demonstrate the opportunities of approaching the stability study of traffic dynamics from systems and control engineering perspective, which may surface interesting physical interpretations.

It is noteworthy to state that dynamics with a single delay is mostly preferred in the literature as a departure point of the stability analysis in the delay domain. This is mainly due to the fact that single delayed dynamics are easier to handle as can be seen in the literature (Olgac, Sipahi 2002, Niculescu 2001, Stepan 1989).

In presence of independent (non-identical) time delays in the dynamics, we demonstrate that, compared to taking into account a single delay for all the sensing delays of the drivers, a) the stability regions may appear in a bounded delay domain, b) a stability region which is independent of one of the delays (unbounded stability regions) may surface, or c) stability cannot be recovered from an unstable non-delayed dynamics no matter how delays are chosen. Our results in a) and b) above are especially of interest due to the fact that larger stability regions correspond to more degree of robustness (Stepan 1989, Hale, Huang 1993, Niculescu 2001, Michiels 2002), which are always desirable under the presence of non-negligible modelling errors.

In all the case studies, we deploy the CTCR ${ }^{2}$, (Olgac, Sipahi 2002, Sipahi, Olgac 2005, Sipahi 2005) in order to arrive in the stability pictures in delay-parameter space. Note that one can also follow a recently developed geometric characterization methodology to reach exactly the same results, $(\mathrm{Gu}$, et al. 2005).

\section{ANALYSIS OF TIME DELAY EFFECTS}

The model proposed in (Helbing 2001) is extended here for the analysis. The objective is to discuss on this continuous-time linear traffic model and, via stability analysis, elaborate the physical features that surface under presence of single and multiple time delays. For this purpose, a conceptual circular traffic is considered, Figure 1 (a configuration usually preferred in the literature, see (Orosz, Stepan 2004) and colleagues' works)

\subsection{Multiple Vehicle Following Strategy with a Single Time Delay}

This subsection deploys a linear time invariant traffic dynamics with a single time delay. It can also be in-

\footnotetext{
2 Cluster Treatment of Characteristic Roots
} 
terpreted as the linearization of a non-linear dynamics with multiple vehicle following strategy at its equilibrium solution, (Sipahi, Niculescu 2006):

$$
\dot{x}_{n}(t)=\sum_{j=1}^{k} \alpha_{n, j}\left(x_{n+j}(t-\tau)-x_{n}(t-\tau)\right)
$$

where $n=6$ vehicles are considered, $k$ an integer which is taken as $k \in[1,4]$ representing how many vehicles ahead are followed by the drivers, and the time delay $\tau \in \mathbf{R}^{+}$. Note on the above equation that $\tau$ represents sensing delay of the drivers. Originally, equations carry acceleration terms on the left hand side and velocity terms on the right hand side of the equality. We just reduce the order of the differential equation by one. The right hand side of the above equation can be seen as a feedback control law of the human drivers. We stress that dynamics in (1), when $\alpha_{n, j}=\beta=0, j>1$, is the simplest representation of traffic dynamics as proposed in 1950 by Reuschel, see (Helbing 2001). In our work, the constant $\alpha_{n, j}$ are taken as $\alpha_{n, 1}=\gamma=1.05, \alpha_{n, j}=\beta=0.8, j>1$. The aim of this section, as mentioned before, is to detect analytically the exact stability regions of the dynamics Eq.(1) in single time-delay domain $\tau$, when $k$ registers various values, $k \in[1,4]$.

2.1.1. Stability analysis results Using the circular path in Figure 1 for $n=6$ vehicles, the characteristic equation of the dynamics, when $k=4$, is given as:

$$
\begin{gathered}
C E(s, \tau)=s^{6}+(18 \beta+6 \gamma) s^{5}\left(e^{-\tau s}\right)+(90 \beta \gamma \\
\left.+15 \gamma^{2}+126 \beta^{2}\right) s^{4}\left(e^{-\tau s}\right)^{2}+\left(20 \gamma^{3}+492 \gamma \beta^{2}\right. \\
\left.+174 \gamma^{2} \beta+428 \beta^{3}\right) s^{3}\left(e^{-\tau s}\right)^{3}+\left(657 \beta^{2} \gamma^{2}\right. \\
\left.+156 \gamma^{3} \beta+15 \gamma^{4}+1158 \beta^{3} \gamma+705 \beta^{4}\right) s^{2}\left(e^{-\tau s}\right)^{4} \\
+\left(450 \beta^{5}+990 \beta^{4} \gamma+798 \beta^{3} \gamma^{2}+6 \gamma^{5}\right. \\
\left.+306 \beta^{2} \gamma^{3}+54 \beta \gamma^{4}\right) s\left(e^{-\tau s}\right)^{5}=0
\end{gathered}
$$

Note that this equation represents the most complicated characteristic equation taken into account, $k=$ 4. The non-delayed dynamics for this case, i.e., when $\tau=0$, possesses six roots; $s_{1}=0, s_{2}=-2 \gamma-$ $2 \beta, s_{3,4}=-3 \beta-3 \gamma / 2 \mp i \gamma \sqrt{3} / 2, s_{5,6}=-5 \beta-$ $\gamma / 2 \mp i \gamma \sqrt{3} / 2$ (with $i=\sqrt{-1}$ ), all of which have negative real parts (since $\gamma>0$ and $\beta>0$ ) except the zero pole (invariant pole) on the origin of the complex plane. This pole represents the rigid body motion of the vehicles travelling around the circular path, i.e., it

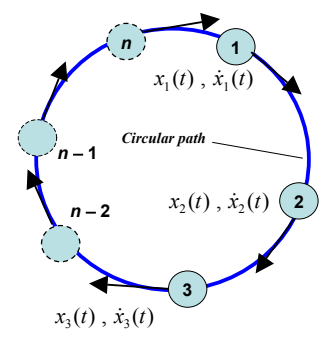

Fig. 1. Circular path scenario with $n$ vehicles traveling.
Table 1. Stability Margin vs. the number of vehicles followed $(k)$.

\begin{tabular}{|c|c|}
\hline $\mathrm{k}$ & Stability Margin, $\tau[\mathrm{sec}] \in$ \\
\hline 1 & {$[0,0.498)$} \\
2 & {$[0,0.349)$} \\
3 & {$[0,0.304)$} \\
4 & {$[0,0.297)$} \\
\hline
\end{tabular}

is the motion mode where there is no relative displacement of vehicles with respect to each other, $k \in[1,4]$. Using the continuity principle argument (Niculescu 2001), it follows that the remaining poles are still in $\mathbf{C}^{-}$for small delay values, $\tau=\epsilon>0$. In conclusion, the computation of the delay margin makes sense.

Remark: The presence of an invariant root in the nondelayed characteristic equation of a time-delayed dynamics may sometimes induce instability by allowing a second root to cross from left to right half of the complex plane, across the origin, for a finite time delay $\tau$. This delay should satisfy concurrently $\left.\frac{d C E(s, \tau)}{d s}\right|_{s=0}=0$ and (2). However, in the characteristic equations $(k \in[1,4])$ we consider here this is never the case, i.e., $\left.\frac{d C E(s, \tau)}{d s}\right|_{s=0} \neq 0$. Thus an instability never occurs by a pole crossing the origin.

In the following, we present the analytical stability analysis results on the dynamics Eq.(1) in $\tau$ domain, i.e., we wish to present the time delay margin in which the dynamics preserves its stability, and next to check if there exists larger delay values for which stability can be recovered.

2.1.2. Physical Interpretations Assuming the time delays influencing the drivers are identical to each other, drivers selecting a strategy of following more than one vehicle ahead of them cause narrower stability margins in the entire traffic dynamics, see Table 1. According to the mathematical model at hand, it is preferable that the drivers follow only the vehicles in front of them, since this selection offers a wider stability margin for the linear stability of the entire dynamics.

With this case study, we also aim to merge systems and control theory into the stability analysis of traffic dynamics. From the analysis, one may surface some unexpected phenomena, such as in the above analysis. Note that, this approach has not been pursued thoroughly in the open literature except a few publications (Orosz, Stepan 2004, Orosz, et al. 2004), and the current work, as to our knowledge, seems to be the first one in that direction.

\subsection{Multiple Vehicle Following with Multiple Time Delays}

In this case study, we compare two different traffic scenarios using an extended version of the dynamics in (1). In the first one, a single vehicle is followed by 
all the drivers with an identical reaction time delay, $\tau$. In the second scenario, all drivers follow the two vehicles ahead of them, however sensing a nearer vehicle is assumed to be faster than a further one, thus there exists two independent time delays, giving rise to a multiple time-delayed dynamics. Note that, all drivers pay more attention to a nearer vehicle in front of them than to a vehicle in further distance; hence such an assumption is quite realistic.

First of all, we take $n=3$ vehicles on the circular path given in Figure 1. Before we proceed, we wish to classify the analysis of this part since we construct the feedback law in three different ways:

Strategy 1 Drivers follow either one or two vehicles ahead of them, by performing only position feedback, i.e.

$$
\ddot{x}_{n}(t)=\sum_{j=1}^{2} \alpha_{n, j}\left(x_{n+j}\left(t-\tau_{j}\right)-x_{n}\left(t-\tau_{j}\right)\right)
$$

Strategy 2 Drivers follow either one or two vehicles ahead of them, by performing only velocity feedback, i.e.

$$
\ddot{x}_{n}(t)=\sum_{j=1}^{2} \alpha_{n, j}\left(\dot{x}_{n+j}\left(t-\tau_{j}\right)-\dot{x}_{n}\left(t-\tau_{j}\right)\right)
$$

Strategy 3 Drivers follow either one or two vehicles ahead of them, by performing a combination of position and velocity feedback, i.e.

$$
\begin{gathered}
\ddot{x}_{n}(t)=\sum_{j=1}^{2}\left(\alpha _ { n , j } \left(x_{n+j}\left(t-\tau_{j}\right)-x_{n}\left(t-\tau_{j}\right)(5)\right.\right. \\
\left.\left.+\dot{x}_{n+j}\left(t-\tau_{j}\right)-\dot{x}_{n}\left(t-\tau_{j}\right)\right)\right)
\end{gathered}
$$

The main objective here is to understand how different control laws under the influence of single and multiple time delays may reveal different stability features of the dynamics, and whether or not the stability would be improved further when multiple car following strategy is selected $\left(\alpha_{n, 2} \neq 0\right)$. Similar to the previous case study, we take $\alpha_{n, 1}=\gamma=1.05$. When a single vehicle is followed by the drivers $\alpha_{n, 2}=\beta=0$, and when two vehicles are followed $\alpha_{n, 2}=\beta=0.8$. In the following we present the results obtained.

2.2.1. Stability analysis results The stability analysis in single and multipme time delay domains will be analyzed by the CTCR technique, (Sipahi, Olgac 2005, Sipahi 2005).

Stability results and physical interpretations for position feedback:

- Following one vehicle ahead: $\left(\alpha_{n, 2}=0\right.$ in Eq.(3)), dynamics is single time-delayed. A stability analysis declares that the dynamics is always unstable no matter what $\tau$ is.

- Following two vehicle ahead: For this case, same result follows as above. Drivers following two cars ahead of them cannot improve the stability of the entire traffic dynamics. The dynamics is always unstable for any selection of time delays, $\tau_{1}$ and $\tau_{2}$. The instability picture, (Figure 2) demonstrates that no stable regions appear in $\tau_{1}$ vs $\tau_{2}$ parametric domain.

Conclusion: Note that using only a position feedback control law makes the non-delayed dynamics unstable, and the presence of time delay is not able to suggest a stability recovery although multiple vehicles are followed by the drivers.

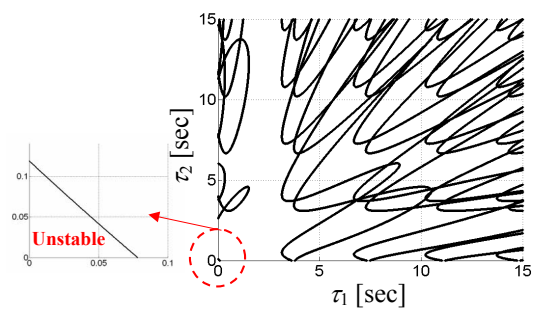

Fig. 2. Instability picture under position feedback, Strategy 1.

Stability results and physical interpretations for velocity feedback:

- Following one vehicle ahead: The dynamics in Eq.(4) becomes single time-delayed. A stability analysis declares the stability margin as $\tau \in$ $[0,0.576) \mathrm{sec}$.

- Following two vehicles ahead: For this case, the dynamics in Eq.(4) is stable in the shaded region given in $\tau_{1}$ vs $\tau_{2}$ domain, Figure 3 . Interestingly, the entire dynamics has an unbounded stability region along $\tau_{2}$ for $\tau_{1}<0.1374 \mathrm{sec}$. Physically, this explains that if drivers can act no later than $\tau_{1}=0.1374 \mathrm{sec}$ for their velocity feedback decisions based on the nearer vehicle, no matter have late they act for their decisions they make based on the velocity of the further $\left(2^{\text {nd }}\right.$ vehicle ahead) vehicle, the traffic dynamics remains stable.

Furthermore, we split the stability region into two using the $45^{\circ}$ dashed red line. We focus on the region where $\tau_{2}>\tau_{1}$, since we assumed that $\tau_{1}$ is smaller than $\tau_{2}$ (sensing a nearer vehicle is less time-delayed compared to sensing a further one). If $0.1374<\tau_{1}<0.5 \mathrm{sec}$, stability independent of $\tau_{2}$ disappears, and decreasing $\tau_{1}$ allows larger $\tau_{2}$ values, or vice versa. Clearly, there exists a trade-off in this picture, and both delays cannot be independently increased without destroying the stability of the dynamics.

Conclusion: We stress that in contrast to position feedback only, velocity feedback control law is preferable since it makes the non-delayed dynamics stable. Moreover, if $\tau_{1}=0$ is selected and a stability analysis is performed, one finds that this dynamics is stable independent of $\tau_{2}$. This suggests that there maybe a $\tau_{1}>0$ beyond which this stable independent of delay 


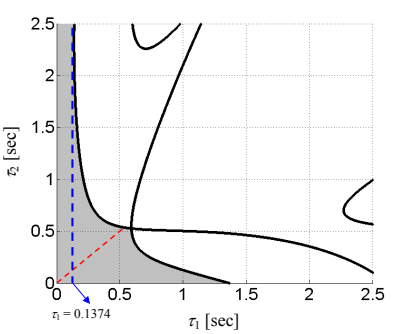

Fig. 3. Stability picture (shaded) under velocity feedback, Strategy 2.

region may not exist (it is only a possibility); which is the case in this analysis.

Stability results and physical interpretations for velocity and position feedback:

- Following one vehicle ahead: Again the dynamics in Eq.(5) becomes single time-delayed. A stability analysis declares that the dynamics is stable for time delays in the range of $\tau \in$ $[0,0.291) \mathrm{sec}$.

- Following two vehicles ahead: For the dynamics in Eq.(5), the stability picture is depicted in Figure 4 in $\tau_{1}$ vs $\tau_{2}$ parametric domain. The shaded region, which is bounded on both axis, represents the stable region of the entire dynamics. In order to designate our assumption $\tau_{2}>\tau_{1}$, we mark the figure with a dashed red line. If drivers can act earlier than $\tau_{1}=0.4 \mathrm{sec}$ for sensing the velocity and position variations between their vehicle and the preceding vehicle, they can have larger stability margins along $\tau_{2}$ for sensing velocity and position variations between their vehicles and the one further ( $2^{\text {nd }}$ vehicle followed). But note that, the stability region is improving along $\tau_{2}$ (i.e., maximum allowable delay along this axis is increasing) while it is getting narrower along $\tau_{1}$, or vice versa. Hence for the given example dynamics, there exists a trade-off between the two delays, similar to the previous example with velocity feedback law only.

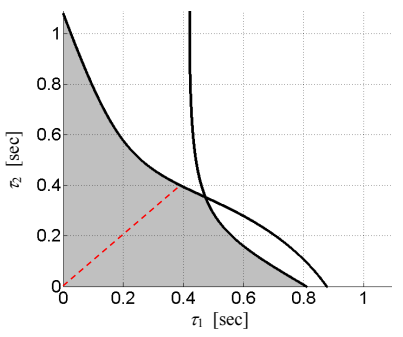

Fig. 4. Stability picture (shaded) under velocity and position feedback, Strategy 3.

Conclusion: It is interesting to see that, compared to only velocity feedback, combination of velocity and position feedback is not suggesting an improvement over the stability regions (compare Figure 3 and Figure 4). Furthermore, the stable independent of $\tau_{2}$ region disappears with the additional position feedback terms in Eq.(5). The stability margin becomes $0 \leq$ $\tau_{2}<1.07 \mathrm{sec}$ when $\tau_{1}=0$. We wish to stress that even one position feedback component is sufficient to destroy this stable independent of $\tau_{2}$ region, i.e., if a position feedback is added to Eq.(4) as follows

$$
\begin{array}{r}
\ddot{x}_{n}(t)=\sum_{j=1}^{2} \alpha_{n, j}\left(\dot{x}_{n+j}\left(t-\tau_{j}\right)-\dot{x}_{n}\left(t-\tau_{j}\right)\right) \\
+\alpha_{n, \nu}\left(x_{n+\nu}\left(t-\tau_{\nu}\right)-x_{n}\left(t-\tau_{\nu}\right)\right)
\end{array}
$$

with $\nu=1,2$. The stability margin when $\tau_{1}=0$ and $\nu=1$ reveals $0 \leq \tau_{2}<1.16 \mathrm{sec}$. If $\nu=2$ is taken, an interesting stability picture surfaces. The dynamics becomes stable in two different intervals of $\tau_{2}$, when $\tau_{1}=0$. These intervals are $0 \leq \tau_{2}<1.347 \mathrm{sec}$ and $4.061 \leq \tau_{2}<4.063 \mathrm{sec}$. We wish to shortly elaborate on this result in the following remark.

Remark: It is very well known that time-delayed dynamics may exhibit more than one stability interval (Olgac, Sipahi 2002, Stepan 1989, Niculescu 2001, $\mathrm{Gu}$, et al. 2005), and this is very advantageous from systems and control engineering perspective since it offers more options in the time-delay domain where the feedback controller can be utilized for stabilization of the dynamics. However, to our best knowledge this phenomenon has neither been recognized in the stability analysis of traffic flow nor it has been constructed for controlling it as a stabilization tool. We reserve further elaboration of this interesting feature for future work.

\subsection{Comments}

Respecting the simplifications of the existing literature, especially by considering a single delay, it is critical to point out the following:

1. As seen from case studies, following multiple vehicles may not be advantageous compared to following the preceding vehicle only. Moreover, a single delay implementation may never offer stability when a position feedback control law is suggested. Larger stability margin may be possible for velocity feedback only as opposed to velocity and position feedback together.

2. Dynamics under certain control law may perform multiple stability intervals as demonstrated over the dynamics in (6) when $\nu=2$. This especially carries importance for controller implementation since it offers more options in the time-delay domain for constructing the controller.

3. Considering more realistic multiple time delays may reveal larger stability regions that may exist in a traffic dynamics. For instance, unbounded stability regions may exist, or stability improvement along one of the delays may require narrower stability margin along the other delay, which can be seen as a trade-off between the time delays. 
4. Multiple delays can be treated as multiple parameter dynamics which could offer more flexibility on how to choose them to achieve stability improvement (stabilization and control synthesis). This study demonstrates there exists research potential that can be pursued on the problem with the integration of systems and controls engineering perspective.

\section{CONCLUSION}

In this work, the stability of some car following traffic dynamics under the influence of time-delays is analyzed. Time delay mainly appears due to the limited sensing and acting capabilities of human drivers. The backbone of the approach is based on system and control engineering perspective. Comparisons between the models with respect to stability improvement in single and multiple delay domains are presented and physical interpretations are given. Via the approach, some interesting features may be obtained in the delay domain; a) more than one stability interval may exist, b) stability independent of one of the delays may surface or c) stability regions may be enhanced.

\section{REFERENCES}

R. W. Allen, T.D. Marcotte, T.J. Rosenthal, B.L. Aponso, "Driver Assesment with Measure of Continuous Control", Proc. $3^{\text {rd }}$ Int. Driving Symp. on Human Factors in Driver Assess., Training and Vehicle Design, Rockport, Maine, USA, 2005.

M. Bando, K. Hasebe, K. Nakanishi, A. Nakayama, "Delay of Vehicle Motion in Traffic Dynamics", Aichi University, 1996, Internal Report.

R.E. Chandler, R. Herman, and E. W. Montroll, "Traffic Dynamics: Analysis of Stability in Car Following. Operational Research", Vol.7, No. 1, pp.165-184, 1958.

M. Green, "'How Long Does It Take to Stop?" Methodological Analysis of Driver PerceptionBrake Times," Transportation Human Factors, vol. 2, pp. 195-216, 2000.

K. Gu, S.-I. Niculescu, and J. Chen, ”On Stability Crossing Curves for General Systems with Two Delays,” J. Math. Anal. Appl., vol. 311, pp. 231253, 2005.

J. K. Hale and S. M. Verduyn Lunel, An Introduction to Functional Differential Equations. New York: Springer-Verlag, 1993.

J. K. Hale and W. Huang, "Global Geometry of the Stable Regions for Two Delay Differential Equations," J. Math. Anal. Appl., vol. 178, pp. 344362, 1993.

D. Helbing, "Traffic and Related Self-Driven ManyParticle Systems," Reviews of Modern Physics, vol. 73, pp. 1067-1141, 2001.
Hoogendoorn, S.P. and Ossen, S., "Parameter Estimation and Analysis of Car-Following Models". In HS Mahmassani (ed.) Flow, Dynamics and $\mathrm{Hu}$ man Interaction (Transportation and Traffic Theory, 16), (pp. 245-266). Elsevier, The Netherlands, 2005.

B. S. Kerner, "Experimental Features of SelfOrganization in Traffic Flow," Physical Review Letters, vol. 81, pp. 3797-3800, 1998.

W. Michiels, Stability and stabilization of time-delay systems, Ph.D. Thesis, KU Leuven, Belgium, May 2002,

S.-I. Niculescu, Delay Effects on Stability, SpringerVerlag, 2001.

T. Nagatani, "Stabilization and Enhancement of Traffic Flow by the Next-Nearest-Neighbor Interaction," Physical Review E, vol. 60, 1999.

A. Nakayama, Y. Sugiyama, and K. Hasebe, "Effect of Looking at the Car That Follows in an Optimal Velocity Model of Traffic Flow," Physical Review E, vol. 65, 2001.

N. Olgac and R. Sipahi, "An Exact Method for the Stability Analysis of Time Delayed Lti Systems," IEEE Trans. Auto. Control, vol. 47, pp. 793-797, 2002.

G. Orosz and G. Stepan, "Hopf bifurcation calculations in delayed systems with translational symmetry", J. Nonlin. Sci., 14 (2004) 505-528.

G. Orosz, R. E. Wilson and B. Krauskopf, "Bifurcations in a Car-Following Model with Delay", IFAC TDS, Leuven, Belgium, 2004.

R. W. Rothery, 'Transportation Research Board (Trb) Special Report 165," in "Traffic Flow Theory", 2nd Edition, N. H. Gartner, C. J. Messner, and A. J. Rathi, Eds., 1998.

R. Sipahi, Cluster Treatment of Characteristic Roots, CTCR, A Unique Methodology for the Complete Stability Robustness Analysis of Linear Time Invariant Multiple Time Delay Systems Against Delay Uncertainties, Ph.D. Thesis, University of Connecticut, Mechanical Engineering Department, August 2005.

R. Sipahi and N. Olgac, "Complete Stability Robustness of Third-Order Lti Multiple Time-Delay Systems," Automatica, vol. 41, pp. 1413-1422, 2005.

R. Sipahi and S.-I. Niculescu, "Some Remarks on the Characterization of Delay Interactions in Deterministic Car Following Models", MTNS Proc., July 2006 in Kyoto (Japan).

G. Stepan, Retarded Dynamical Systems: Stability and Characteristic Function. Longman, New York, US, 1989.

M. Treiber, A. Hennecke, and D. Helbing, "Congested traffic states in empirical observations and microscopic simulations", Physical review E, vol.62, pp. 1805-1824, 2000.

M. Treiber, A. Kesting and D. Helbing, 'Delays, Inaccuracies and Anticipation in Microscopic Traffic Models”, Physica A, Volume 360, 71-88 (2006). 